ARTICLE

Received 2 Dec 2013 | Accepted 11 Apr 2014 | Published 19 May 2014 DOl: 10.1038/ncomms4863

\title{
Cold ablation driven by localized forces in alkali halides
}

Masaki Hada ${ }^{1, \star, \dagger}$, Dongfang Zhang ${ }^{1, \star}$, Kostyantyn Pichugin ${ }^{1, \dagger}$, Julian Hirscht ${ }^{1}$, Michał A. Kochman ${ }^{1,2}$, Stuart A. Hayes ${ }^{1}$, Stephanie Manz ${ }^{1}$, Regis Y.N. Gengler ${ }^{1}$, Derek A. Wann ${ }^{2} \dagger$, Toshio Seki ${ }^{3}$, Gustavo Moriena ${ }^{1,4}$, Carole A. Morrison ${ }^{2}$, Jiro Matsuo ${ }^{3}$, Germán Sciaini ${ }^{1, \dagger} \&$ R.J. Dwayne Miller ${ }^{1,4}$

Laser ablation has been widely used for a variety of applications. Since the mechanisms for ablation are strongly dependent on the photoexcitation level, so called cold material processing has relied on the use of high-peak-power laser fluences for which nonthermal processes become dominant; often reaching the universal threshold for plasma formation of $\sim 1 \mathrm{~J} \mathrm{~cm}^{-2}$ in most solids. Here we show single-shot time-resolved femtosecond electron diffraction, femtosecond optical reflectivity and ion detection experiments to study the evolution of the ablation process that follows femtosecond $400 \mathrm{~nm}$ laser excitation in crystalline sodium chloride, caesium iodide and potassium iodide. The phenomenon in this class of materials occurs well below the threshold for plasma formation and even below the melting point. The results reveal fast electronic and localized structural changes that lead to the ejection of particulates and the formation of micron-deep craters, reflecting the very nature of the strong repulsive forces at play.

\footnotetext{
${ }^{1}$ The Max Planck Institute for the Structure and Dynamics of Matter, and The Hamburg Centre for Ultrafast Imaging, Luruper Chaussee 149, 22761 Hamburg, Germany. ${ }^{2}$ School of Chemistry, University of Edinburgh, West Mains Road, Edinburgh EH9 3JJ, Scotland. ${ }^{3}$ Quantum Science and Engineering Center, Kyoto University, Gokasho, Uji, Kyoto 611-0011, Japan. ${ }^{4}$ Departments of Chemistry and Physics, University of Toronto, 80 St George St, Toronto, Ontario, Canada M5S3H6. * These authors contributed equally to this work. †Present addresses: Materials \& Structures Laboratory, Tokyo Institute of Technology, Yokohama 226-8503, Japan and JST-PRESTO, Kawaguchi 332-0012, Japan (M.H.); Department of Chemistry, University of Waterloo, Waterloo, Ontario, Canada N2L 3 G1 (K.P. and G.S.); Department of Chemistry, University of York, Heslington, York YO10 5DD, UK (D.A.W.). Correspondence and requests for materials should be addressed to R.J.D.M. (email: dwayne.miller@mpsd.mpg.de).
} 
aser ablation involves many-body interactions which, in a collective response to optical excitation, lead to material removal. The ablation processes in metals, semiconductors and dielectrics are different owing to the nature of their electronic and structural properties. In the case of semiconductors and metals exposed to femtosecond ( $\mathrm{fs}$ ) laser irradiation, different absorption energy regimes have been observed ${ }^{1-4}$. At relatively low incident laser fluence $\left(F<100 \mathrm{~mJ} \mathrm{~cm}^{-2}\right)$, the solid surface is heated up and the material thermally evaporates or sublimes on the nanosecond-to-microsecond timescale. At higher laser peak powers, providing $F$ is in the range of $300-500 \mathrm{~m} \mathrm{~cm}^{-2}$, the system is often pushed into a metastable liquid phase as a result of rapid heating and melting. In such a metastable state, homogeneous bubble nucleation leads to the removal of large fragments of the material through processes known as spallation, phase explosion and fragmentation ${ }^{5,6}$. With even higher $F$ $\left(>1 \mathrm{~J} \mathrm{~cm}^{-2}\right)$, the solid surface changes directly into a gaseous plasma via multiphoton ionization. This often leads to the ejection of charged fragments due to strong space-charge effects ${ }^{7}$. This latter process, which involves states that are unreachable by any possible thermal pathway, is commonly referred to as 'cold ablation' and relies on effective potential energy channelling into translational degrees of freedom.

Due to their much wider bandgap $\left(E_{\mathrm{g}}\right)$, fs laser interaction with dielectrics often involves multiphoton absorption processes. If $E_{\mathrm{g}}$ largely exceeds the photon energy $(h v)$, absorption is frequently dominated by highly nonlinear processes that lead to ionization and avalanche effects ${ }^{8-10}$. The investigation of elementary electron-hole pairs (excitons) in ionic dielectric materials, such as alkali halides, has therefore required the use of $h v$ in the ultraviolet (UV) range to mitigate the aforementioned competing channels. The effects of UV light on the electronic properties of alkali halides were discovered in 1896 when initially colourless crystals were found to gain colour on irradiation ${ }^{11}$. Since then, several studies have been performed to understand the formation of such long-lived colour centres ${ }^{12}$. By analogy to gas phase studies ${ }^{13}$, the observed absorption band in alkali halides was initially attributed to the electron back transfer from the anion to a neighbouring cation. In this case, the cation would gain neutral character and thus absorb light in the visible range of the spectrum. The scenario in the crystalline phase was found to be considerably more complicated than that for contact ion pairs in vacuum, with different species originating from a highly unstable primary exciton. Nevertheless, the highly localized nature of the excited state in alkali halides made these materials an ideal target for the investigation of self-trapped excitons, self-trapped holes and different transient precursors of long-lived lattice defects and vacancies responsible for the chromatic effect ${ }^{14-21}$. Moreover, several experiments involving time-resolved ion detection have been performed to investigate the stability of alkali halide surfaces following fs laser irradiation ${ }^{22-25}$ at moderate $F \sim 1-10 \mathrm{~mJ} \mathrm{~cm}^{-2}$ or low-energy electron irradiation ${ }^{26}$. These results have connected the states of alkali halides irradiated by fs laser pulses or electron beams with surface desorption mechanisms. In these studies, surface metallization ${ }^{25}$ and nonthermal layer-bylayer ${ }^{26}$ desorption processes, driven by the formation of excitons, have been proposed. On the other extreme, the ablation process due to Columbic explosion induced by multiphoton ionization of the sample's surface has also been discussed for dielectric materials at very high photoexcitation levels ${ }^{27,28}, F>1 \mathrm{~J} \mathrm{~cm}^{-2}$.

In this report, we investigate the mechanism and driving force of the ablation process that follows 2 or 3 photon fs laser excitation of alkali halides under the intermediate range of photoexcitation $F \sim 100 \mathrm{~mJ} \mathrm{~cm}^{-2}$. This range has not been fully explored and it is critical to separate novel electronic factors related to Pauli repulsion forces from multiphoton ionization effects. We performed single-shot fs electron diffraction (FED) to track the structural changes associated with laser-driven lattice disruption in this critical fluence regime. This information is complemented by fs optical reflectivity to fully resolve the dynamics and by ion detection studies to link bulk structural and electronic changes to the nature of the ablation process. Our results provide clear evidence of the fast optical and structural changes in alkali halide materials, indicating the development of a lattice-disordering process followed by the expulsion of large clusters and/or fragments. We found that fragmentation remains quite significant even at the moderate laser fluences. For instance, half-a-micron-deep craters are formed in polycrystalline KI sample using single $400 \mathrm{~nm}$ pulses with a fluence value of $60 \mathrm{~mJ} \mathrm{~cm}-2$. The amount of absorbed energy $(2-4 \%$ of the incident) is insufficient in this case to melt the sample, confirming the nonthermal character of the ablation mechanism.

\section{Results}

Fs optical reflectivity and ion detection in silicon. We present our results alongside time-dependent reflectivity changes and ion detection measurements obtained for $\mathrm{Si}$, chosen as a reference system because its fs laser melting and ablation processes are well characterized $^{29-35}$. Thermal melting for $\mathrm{Si}$ is expected to occur for $F$ in the range of $35-70 \mathrm{~mJ} \mathrm{~cm}{ }^{-2}$, which shows up as an increase in reflectivity during the first $\sim 5$ ps (black traces in Fig. 1a) owing to the onset of metallic character caused by the formation of liquid $\mathrm{Si}^{36}$. Laser fluences of $70-100 \mathrm{~mJ} \mathrm{~cm}^{-2}$ correspond to about $9 \%$ of valence carrier excitation and are known to trigger a much faster nonthermal disordering process that takes place on a timescale of $200-400 \mathrm{fs}^{36-41}$. This effect correlates with a very sharp increase in reflectivity observed in the sub-500 fs temporal span (red trace in Fig. 1a). The decrease in reflectivity occurring on the $100 \mathrm{ps}$ timescale is due to surface removal caused by ablation processes that correspond to the spallation, phase explosion and fragmentation of the material. A similar response, which is more pronounced in terms of reflectivity changes, is observed ${ }^{42-44}$ for $F$ higher than $200 \mathrm{~mJ} \mathrm{~cm}^{-2}$ (see blue and magenta traces in Fig. 1a). This latter observation correlates with the first ablation threshold, $F_{\text {abl }}$, determined in our ion detection measurements (see Fig. 2a). For $F>F_{\mathrm{abl}} \approx 200 \mathrm{~mJ} \mathrm{~cm}^{-2}$, the detection of positively charged fragments from $\mathrm{Si}$ is evident. There is in general good agreement between our time-resolved reflectivity and ion detection measurements, and previous studies ${ }^{30}$ on laserinduced surface disordering of $\mathrm{Si}$. As shown in Fig. 2a, increasing the incident fluence above $800 \mathrm{~mJ} \mathrm{~cm}^{-2}$ results in a dramatic enhancement of the ion yield. This new threshold fluence, $F_{\text {plasma, was attributed to the formation of gaseous }}$ plasma. According to the literature ${ }^{44}$, this process becomes dominant at $F>1 \mathrm{~J} \mathrm{~cm}^{-2}$. In summary, we have found, through ion detection measurements, two ablation thresholds $\left(F_{\mathrm{abl}} \approx 200 \mathrm{~mJ} \mathrm{~cm}{ }^{-2}\right.$ and $\left.F_{\text {plasma }} \approx 800 \mathrm{~mJ} \mathrm{~cm}^{-2}\right)$ for $\mathrm{Si}$ that concur with previous findings $34,35,42$.

Fs optical reflectivity and ion detection in alkali halides. Owing to the wide bandgaps of $\mathrm{CsI}(6.1 \mathrm{eV}), \mathrm{NaCl}(7.6 \mathrm{eV})$ and $\mathrm{KI}$ $(6.0 \mathrm{eV})$ crystals, photoexcitation with $400-\mathrm{nm}$ wavelength light $(3.1 \mathrm{eV})$ requires two or three photons for above bandgap excitation and the creation of electron-hole pairs. The timedependent changes in reflectivity for $\mathrm{CsI}$ and $\mathrm{NaCl}$ single crystals and polycrystalline KI films, presented as black and red curves in the Fig. $1 \mathrm{~b}-\mathrm{d}$ for the range of modest $F$, exhibit a sharp subpicosecond decay in amplitude, followed by a partial recovery with several picosecond rise times. This trend is clearly different from the rapid enhancement in reflectivity followed by its 

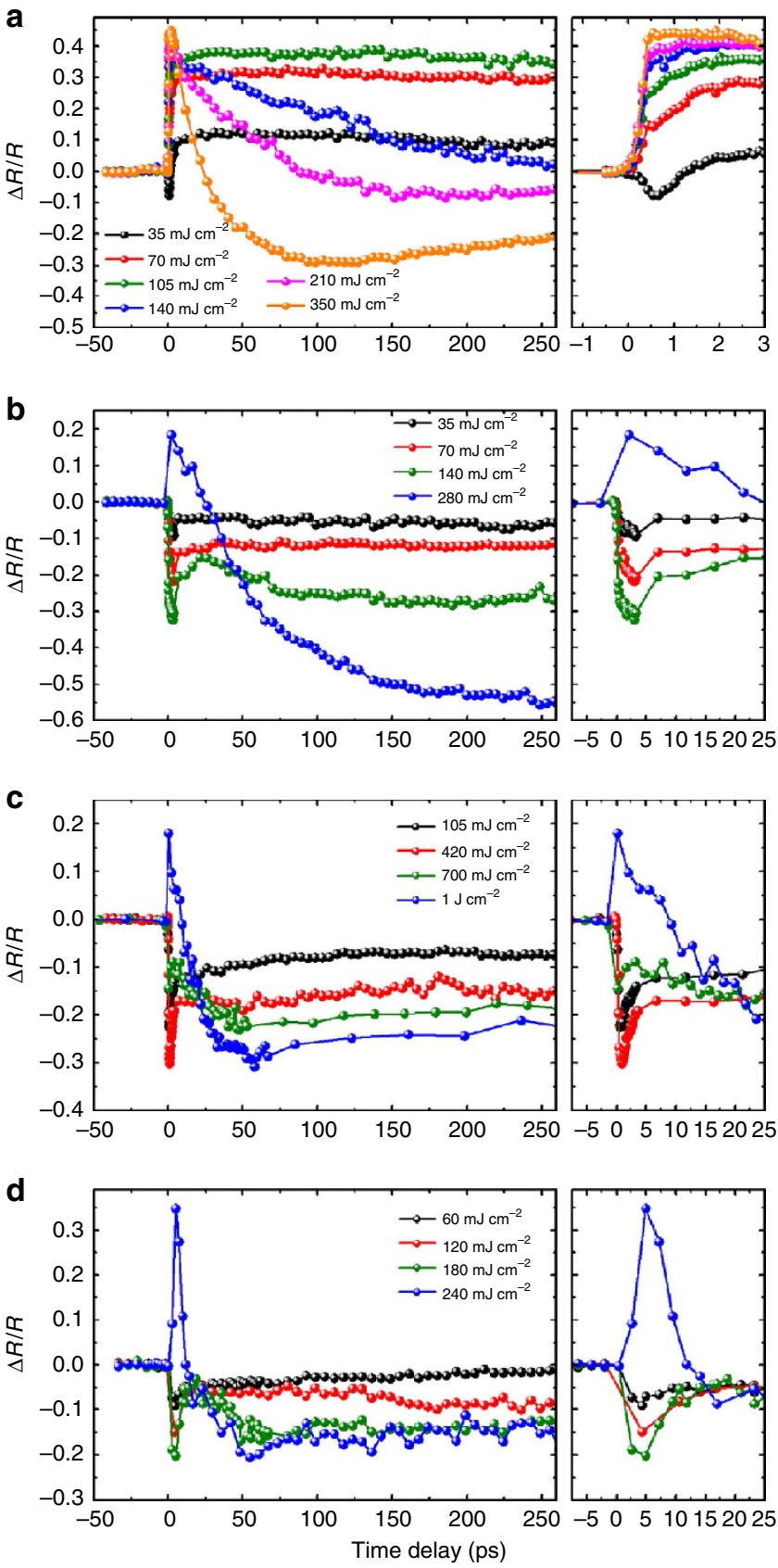

Figure 1 | Time-dependent reflectivity changes. Relative reflectivity changes as a function of the time delay (a) for $\mathrm{Si}$, (b) for Csl, (c) for $\mathrm{NaCl}$ and (d) for $\mathrm{KI}$. The incident laser fluences corresponding to the each plot are indicated in the figures.

subsequent decay within 100-200 ps observed for the Si (Fig. 1a) under similar photoexcitation conditions. At $F \approx 140,700$ and $180 \mathrm{~mJ} \mathrm{~cm}^{-2}$, for Fig. 1b-d, a small bump appears at the end of the well in the green traces shown for CsI, $\mathrm{NaCl}$ and $\mathrm{KI}$, respectively. Going to even higher fluences makes this feature grow (blue traces in Fig. 1b-d) into a large peak closely resembling that observed during the formation of liquid Si. On the basis of the fact that the reflectivity of ionic and glass materials increases as they melt ${ }^{45}$, the latter result provides strong evidence for melting occurring at the crystal's surface. Therefore, we can consider those values of $F, 140,700$ and $180 \mathrm{~mJ} \mathrm{~cm}^{-2}$, as an estimate for the onset of thermal melting, $F_{\text {melt }}$, for $\mathrm{CsI}, \mathrm{NaCl}$ and KI samples, respectively. On the other hand, it is safe to assume a
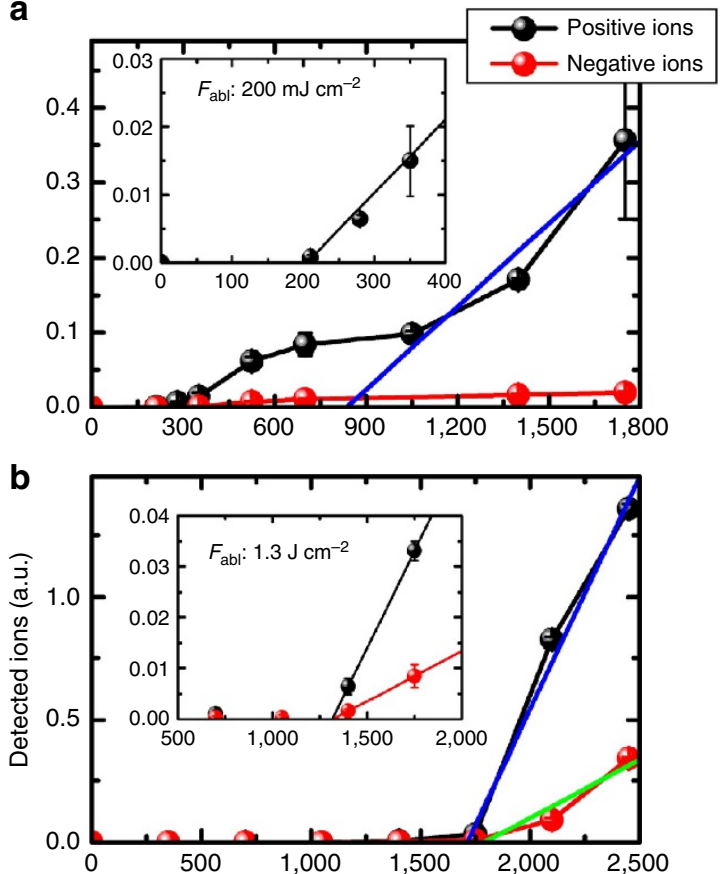

C

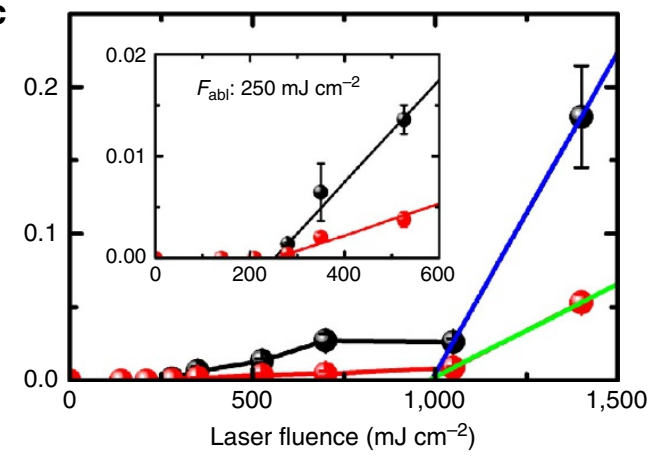

Figure 2 | Ablation ion yields as a function of laser fluence. Laser fluencedependent yields of positive (black trace) and negative (red trace) ions measured using a simple homemade ion detection system (a) for $\mathrm{Si}$, (b) for $\mathrm{NaCl}$ and (c) for $\mathrm{Csl}$ single-crystalline samples. Insets show magnified views near the first detected ablation threshold, $F_{a b l}$, for each sample. The second threshold, $F_{\text {plasmar }}$ is determined as the intersection of the blue and/or green lines with the abscissa. The difference in the yield of negative and positive ions reflects the fact that, in general, cations are more thermodynamically stable species than their corresponding anions.

by inspecting the red traces in Fig. 1b-d that no melting happens for the corresponding alkali halides at and below the 70,420 and $120 \mathrm{~mJ} \mathrm{~cm}^{-2}$ fluences.

The ion detection measurements for $\mathrm{NaCl}$ and $\mathrm{CsI}$ samples are summarized in Fig. 2b,c. Through careful examination of the CsI data, we also found a two-threshold behaviour with $F$ values of $\approx 250 \mathrm{~mJ} \mathrm{~cm}^{-2}$ and $\approx 1 \mathrm{~J} \mathrm{~cm}^{-2}$ for ion detection, which are higher than $F_{\text {melt }}$. In direct comparison with the Si data, the former threshold can be related to spallation, phase explosion and fragmentation processes. The latter threshold is assigned to gaseous plasma formation. In the case of $\mathrm{NaCl}$, these two thresholds are very close, $F_{\mathrm{abl}} \approx 1.3 \mathrm{~J} \mathrm{~cm}^{-2}$ and $F_{\text {plasma }} \approx 1.7 \mathrm{~J} \mathrm{~cm}^{-2}$, suggesting that both processes might occur near-simultaneously or that ionization effects leading to plasma formation are simply dominant due to its relatively higher $\frac{E_{\mathrm{g}}}{h v}$ ratio. 


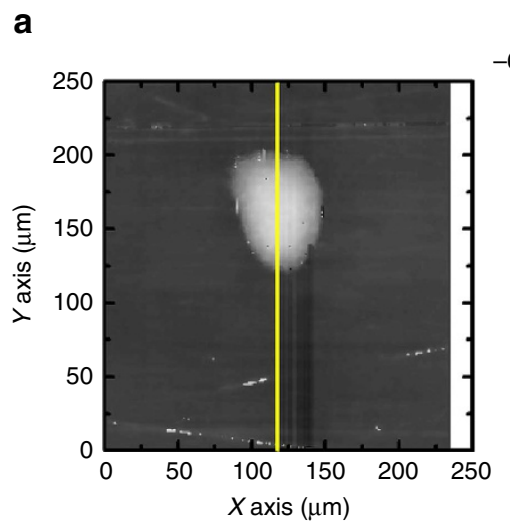

Depth $(\mathrm{nm})$

$-600-300 \quad 0$
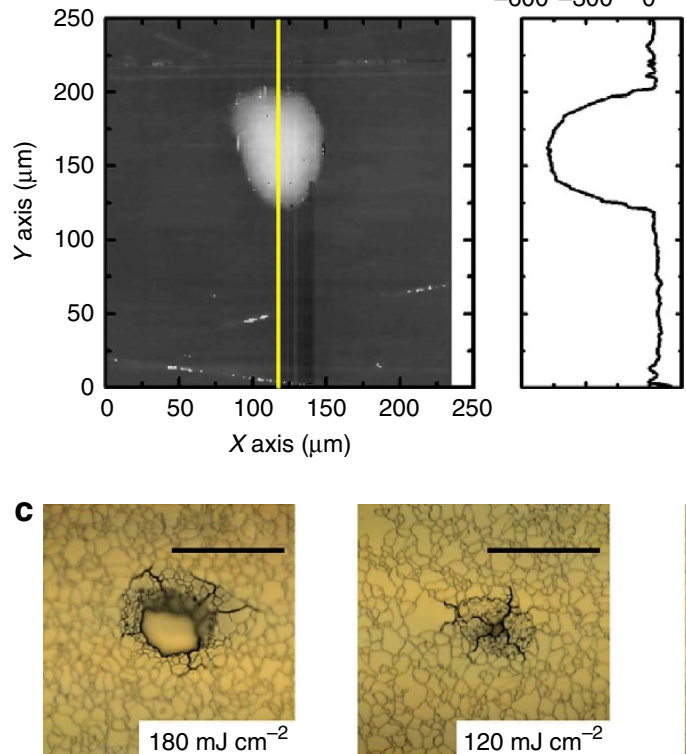

b

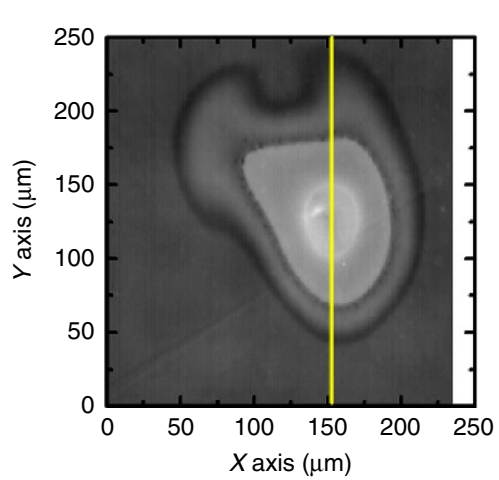

Depth $(\mathrm{nm})$
$-30-20-10 \quad 0 \quad 10$

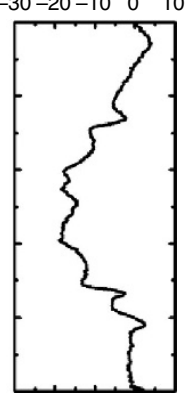

Figure 3 | Crater measurements. All craters were produced by single $400 \mathrm{~nm}$ pump pulses. Micrographs of the craters alongside their 2D sectional profiles for single-crystalline $\mathrm{Csl}$ (a) and single-crystalline $\mathrm{Si}(\mathbf{b})$; incident fluences were 140 and $350 \mathrm{~mJ} \mathrm{~cm}^{-2}$, respectively. (c) Craters generated in $2.5-\mu \mathrm{m}$-thick polycrystalline KI films at $F \approx 60-180 \mathrm{~mJ} \mathrm{~cm}^{-2}$ (scale bar, $100 \mu \mathrm{m}$ ). (d) Representative profile measurements for the crater shown in $\mathbf{c}$, $F \approx 60 \mathrm{~mJ} \mathrm{~cm}^{-2}$.

\begin{tabular}{|c|c|c|}
\hline & $F, \mathrm{~mJ} \mathrm{~cm}^{-2}$ & Depth, nm \\
\hline \multirow[t]{3}{*}{$\mathrm{Si}$} & 350 & 26 \\
\hline & 210 & 16 \\
\hline & 70 & 9 \\
\hline \multirow[t]{2}{*}{$\mathrm{NaCl}$} & 420 & 970 \\
\hline & 350 & 950 \\
\hline \multirow[t]{3}{*}{ Csl } & 280 & 704 \\
\hline & 140 & 472 \\
\hline & 105 & 418 \\
\hline \multirow[t]{3}{*}{$\mathrm{KI}$} & 180 & $>2,500$ \\
\hline & 120 & 1,400 \\
\hline & 60 & 500 \\
\hline
\end{tabular}

Crater depths were determined using the following techniques: interferometry for $\mathrm{Cs}$ and $\mathrm{Si}$, confocal laser microscopy for $\mathrm{NaCl}$ and the contact stylus profilometery for $\mathrm{KI}$.

Crater depth measurements. Figure 3a,b shows typical crater shapes observed via optical interferometry after exposing CsI and $\mathrm{Si}$ samples to single-excitation pulses with $F=140$ and $350 \mathrm{~mJ} \mathrm{~cm}^{-2}$, respectively. The crater profiles in KI (Fig. 3c) and $\mathrm{NaCl}$ samples were measured with a stylus contact profilometer and confocal laser microscopy. A brief summary of the crater depths obtained for each sample with various laser fluences is presented in Table 1. Overall, KI samples retain the highest crater depth among all studied alkali halides, with its value being nearly 100 times larger than that in $\mathrm{Si}$ owing to the fundamental difference between fs laser ablation of semiconductors and dielectric materials ${ }^{9}$. We would like to emphasize here that the result of producing larger craters in KI when compared with CsI and $\mathrm{NaCl}$ is readily anticipated. Polycrystalline samples are composed of small crystallites with weaker inter-crystallite forces, and therefore are softer than the single-crystalline counterpart. What is interesting though are the unusually deep $(\approx 0.5 \mu \mathrm{m})$ craters created in alkali halides using single laser pulses with $F<F_{\text {melt }}<F_{\text {abl }}<\mathrm{F}_{\text {plasma }}$. For comparison, making a similar size crater in $\mathrm{Al}_{2} \mathrm{O}_{3}$ with a single laser shot requires nearly two orders of magnitude higher flux with $F=11.3 \mathrm{~J} \mathrm{~cm}^{-2}$ for $395 \mathrm{~nm} \mathrm{light}^{46}$. Another important point is the mechanism responsible for the material expulsion. It is definitely nonthermal in nature, since according to our results no sample melting should happen.

It has been proposed that nonthermal fs laser ablation in dielectric materials proceeds through surface charging, followed by impulsive Coulomb explosion ${ }^{28}$. However, the experimental conditions in this work are significantly different from those in the aforementioned studies for single-shot ablation $(800 \mathrm{~nm}$, $200 \mathrm{fs}, 5 \mathrm{~J} \mathrm{~cm}^{-2}$ ). We cannot therefore explain our findings for large crater formation in alkali halides with this mechanism, as the low laser fluences used in the current experiments are far insufficient ( $F \ll F_{\text {plasma }}$ ) to generate the required charge density for Coulomb explosion to take off.

FED measurements. To further elaborate on the nonthermal mechanism of the ablation process, we performed single-shot FED experiments providing direct structural information on the evolution of crystalline order following fs photoexcitation. Figure 4 shows the time-dependent diffraction intensities for the (020) and (022) Bragg reflections from 50-nm-thick $\langle 100\rangle$ oriented KI films photoexcited with $400 \mathrm{~nm}$ light. The red and blue traces $\left(F=140 \mathrm{~mJ} \mathrm{~cm}^{-2}\right)$ in Fig. 4 reveal the decrease in the crystalline order during the first few tens of picosecond, which then recovers gradually on the nanosecond timescale. The electron diffraction data can be used to estimate the lattice temperature of the KI sample after fs laser irradiation. Accordingly, the recorded intensity signal from diffracted electrons $(I)$ can be expressed by the following equations:

$$
I=|F(\mathbf{K})|^{2}
$$

and

$$
F(\mathbf{K})=G(\mathbf{K}) \sum_{j=1}^{M} f_{j}(\mathbf{K}) D_{j}(\mathbf{K}) \exp \left(2 \pi i \mathbf{K} \cdot \mathbf{r}_{j}\right)
$$




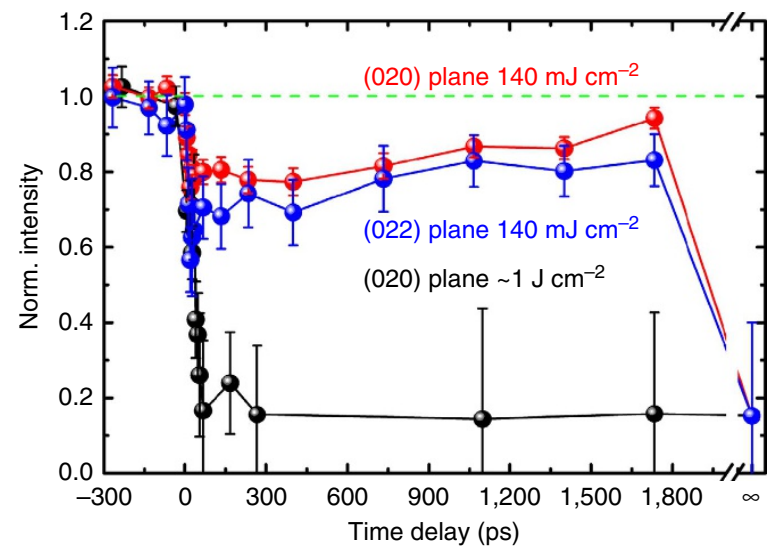

Figure 4 | FED measurements. Time-dependent changes in electron diffraction intensity for $\mathrm{KI}$ under non-reversible, single-shot photoexcitation conditions. Red and blue traces correspond to the relative changes observed for (020) and (022) Bragg reflections, respectively, at $F \approx 140 \mathrm{~mJ} \mathrm{~cm}^{-2}$. Black trace corresponds to relative changes for the $(020)$ reflection at $F \approx 1 \mathrm{~J} \mathrm{~cm}-2$. Vertical error bars represent one s.d. from the mean values for the changes in electron diffraction intensity. Due to irreversible sample damage, the statistics is limited to either 8-10 (blue and red data points) or 5 (black and infinity time data points) single-shot measurements. The green dashed line is a no-change-in-diffractionintensity guide to the eye. Norm., normalized.

Where $\mathbf{K}$ is the scattering vector, $F(\mathbf{K})$ is the structure factor, $G(\mathbf{K})$ is the Laue function, $f_{j}(\mathbf{K})$ is the atomic scattering factor, $D_{j}(\mathbf{K})$ is the Debye-Waller factor and $\mathbf{r}_{j}$ is the equilibrium position of the $j$ th atom in the unit cell. The Debye-Waller factor can be written in terms of temperature-dependent $B$-factors $\left(B_{j}\right)$ or through the mean square displacement $\left(U_{j}\right)$ as

$$
\begin{aligned}
D_{j}(\mathbf{K}) & =\exp \left(-K^{2} B_{j}\right)=\exp \left(-8 \pi^{2} K^{2} U_{j}\right) \\
& =\exp \left[-\frac{8 \pi^{2} U_{j} \sin ^{2} \theta}{\lambda^{2}}\right]
\end{aligned}
$$

where $\theta$ is the scattering angle and $\lambda$ is the de Broglie wavelength of the electrons. We performed a temperature analysis based on values of $B$-factors obtained from the parameterization in ref. 47 derived from the experimental phonon density of states data (see Supplementary Note 1 for more details about these calculations). Assuming the largest intensity change of 25 and $45 \%$ for the (020) and (022) Bragg reflections from $140 \mathrm{~mJ} \mathrm{~cm}^{-2}$ data in Fig. 4, the predicted lattice temperature is $\approx 1,200 \mathrm{~K}$ (kinematic diffraction theory) or $\approx 1,800 \mathrm{~K}$ (with corrections from dynamical diffraction theory ${ }^{48}$ ). These lattice temperatures are certainly above the melting point, and therefore fall off the validity range of the Debye-Waller model, which is only applicable if the unit cell structure of the crystalline material remains intact. After the slow recovery of the signal (time delays of $\approx 1-2 \mathrm{~ns}$ ), Debye-Waller analysis predicts a $\mathrm{T}_{1}=725 \pm 110 \mathrm{~K}$ that is below the melting point of the salt, $954 \mathrm{~K}$. It is worth mentioning that by comparison (see Supplementary Figs 1 and 2), 100-nm-thick KI films show a much stronger intensity decay than those observed for 50-nm-thick films. A similar attenuation effect on the electron diffraction intensity for all diffraction orders was found to be dependent on the material thickness ${ }^{49}$. Accounting for this effect in the analysis results in a smaller temperature increase. Therefore, the observed drop in intensity for the (000)-order electron beam implies that our predicted increase in lattice temperature is indeed an upper estimate. Despite the limitations of the analysis of the FED data, the above findings support the fact that sample heating is insufficient by itself to explain the observed changes in the diffraction, suggesting there are other processes related to the loss and recovery of crystallinity that are occurring in parallel.

\section{Discussion}

One of the possible mechanisms leading to the lattice damage is the formation of point defects in the crystal from selftrapped excitons ${ }^{20}$. The effect of vacancy concentrations on diffraction signal from nanocrystalline materials was previously investigated $^{50}$. On the basis of those predictions, it would require more than $20 \%\left(\approx 2 \times 10^{21} \mathrm{~cm}^{-3}\right)$ lattice point defects to decrease the (020) diffraction intensity by $25 \%$. Such high concentration of point defects is not reachable in our study, which is evident from the optical measurements showing that there are $<10 \%$ photoexcited halide centres at $F=140 \mathrm{~mJ} \mathrm{~cm}^{-2}$ (see Supplementary Note 2 for the details). The other viable causes for the loss of crystallinity are partial local disordering or melting. Referring to the optical reflectivity measurements in KI, $F=140 \mathrm{~mJ} \mathrm{~cm}{ }^{-2}$ falls into the $120-180 \mathrm{~mJ} \mathrm{~cm}^{-2}$ interval that loosely defines the melting threshold. As a result, when the incidence laser fluence is in close proximity to that of $F_{\text {melt }}$, there is a high probability that a fraction of the sample locally becomes either significantly disordered or turns into a liquid. Most likely, in addition to the sample heating, local disordering is the key factor responsible for the reduction in diffraction signal. As the temperature of the sample starts equilibrating within the photoexcited volume, these highly disordered regions attempt to recrystallize, thus contributing to the slow recovery in the diffraction intensity observed in a nanosecond time interval. The latter conclusions are also supported by time-resolved diffraction data at $F=1 \mathrm{~J} \mathrm{~cm}^{-2}$ (shown in Fig. 4). No signal recovery is detected for the plasma-driven damage in $\mathrm{KI}$ at this level of fluence.

Following energy conservation, the increase in lattice temperature after photoexcitation can also be estimated as

$$
\Delta T_{1}=\frac{A_{\mathrm{eff}} F}{\rho \cdot C \cdot f_{\mathrm{t}}}
$$

where $A_{\text {eff }}$ is the effective absorbed fluence fraction, $f_{\mathrm{t}}$ is the film thickness $\sim 50 \mathrm{~nm}, \rho$ and $C$ are the material's density $\left(3.12 \mathrm{~g} \mathrm{~cm}^{-3}\right)$ and specific heat capacity $\left(313 \mathrm{~J} \mathrm{~kg}^{-1} \mathrm{~K}^{-1}\right)$ for $\mathrm{KI}$, respectively. In a very conservative scenario that considers $A_{\text {eff }} \leq 4 \%$ (see Supplementary Fig. 3c), equation (4) provides $\Delta T_{1} \leq 1,150 \mathrm{~K}$. Since the sample was originally at room temperature, the lattice temperature after thermal equilibration therefore results in $T_{1}^{\mathrm{eq}}\left(F=140 \mathrm{~mJ} \mathrm{~cm}^{-2}\right) \leq 1,440 \mathrm{~K}$. Despite the fact that the calculated value of $T_{1}^{\mathrm{eq}}$ is an upper estimate, crater formation in KI was found to occur at $F$ as low as $60 \mathrm{~mJ} \mathrm{~cm}-2$ (see Table 1 and Fig. 3c) for which equation (4) would predict $T_{1}^{\mathrm{eq}}$ $\left(F=60 \mathrm{~mJ} \mathrm{~cm}^{-2}\right)<780 \mathrm{~K}$, which is below the melting point. A similar conclusion can be drawn for $\mathrm{NaCl}$ modifying equation (4) by replacing $f_{\mathrm{t}}$ by $d_{\mathrm{c}}$ (the crater depth) and $A_{\text {eff }}$ by the absorbed fraction measured across the 2-mm-thick bulk single crystal. Hence, a simple thermodynamic calculation based on upper estimates for the absorbed energy, which is in agreement with the values of $F_{\text {melt }}$ determined via fs optical reflectivity measurements, provides strong evidence for nonthermal pathways to be the dominant driving force for the ejection of particulates.

The forces involved can be understood through a simple analogy with contact ion pairs in the gas phase (Fig. 5a). Optical excitation induces localized changes in the electronic distribution, that is, strong electronic stress ${ }^{51-55}$ that provides the initial kick for the consequent material ejection. This latter explanation is consistent with the observation of strain waves ${ }^{56}$ in the $\mathrm{GHz}$ range (see oscillatory behaviour in fs optical reflectivity data shown in Supplementary Fig. 4). A strongly localized nature of 
the excited state is also supported by the FED data (red and blue traces in Fig. 4), which give evidence for the creation of disordered regions that release their energy to the surrounding unexcited crystal (recovery of diffraction signal on sub-ns timescale). At systematically higher fluences necessary to invoke

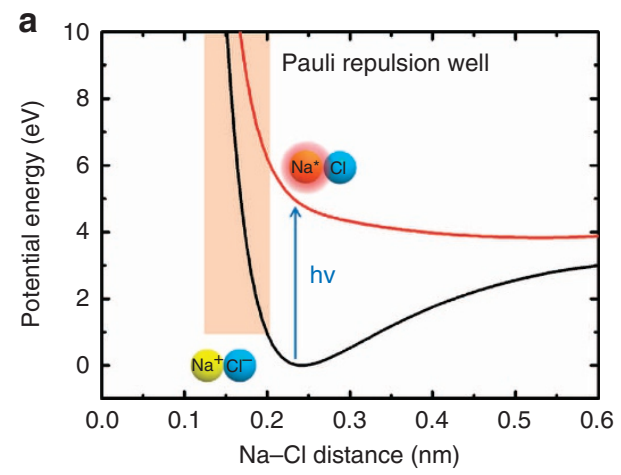

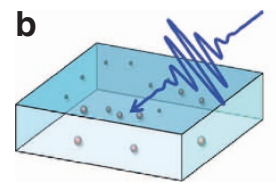

0 ps

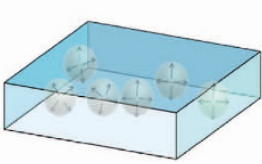

$\sim 10$ ps

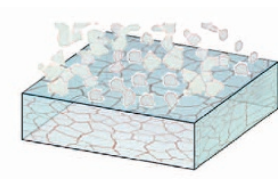

ns $-\mu \mathrm{s}$
Figure 5 | Summary of cold ablation process. (a) Calculated potential energy curves for the $\mathrm{NaCl}$ molecule. The ionic ground and neutral excited states are plotted as black and red curves, respectively (for more details see Computational method Section). Vertical excitation brings the system right to the onset of the Pauli repulsion well where short-range core-core repulsion forces are dominant. The core-core repulsion forces are also expected to be one of the key factors responsible for the material expulsion in alkali halide crystals ${ }^{68}$. (b) A pictorial representation of the cold ablation process. Similar to the $\mathrm{NaCl}$ molecule, photoexcitation in alkali halide crystals causes a local change in the electronic density around the excited halide anions and their neighbouring alkali cations, thus unleashing repulsive forces between partially neutralized atoms. To release the stress, pertinent atoms move from their ground state equilibrium positions and form disordered regions within several picoseconds after the photoexcitation. Further relaxation of the lattice, subject to the amount of generated strain, might involve cracking and ejection of the material on the nanosecond-to-microsecond timescales.
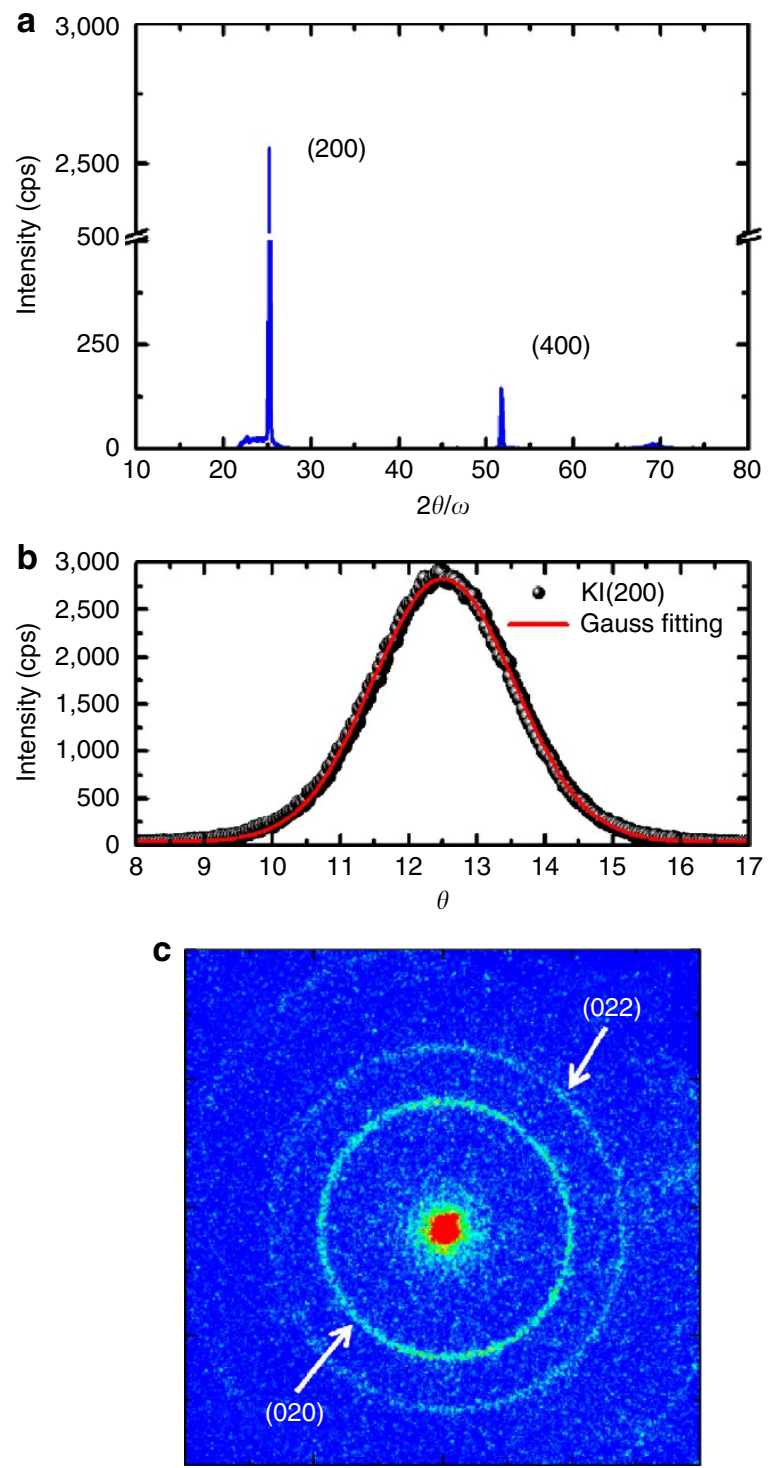

Figure 7 | Characterization of KI thin film crystallinity. (a) X-ray diffraction spectrum from $\mathrm{KI}$ samples. (b) $\mathrm{X}$-ray rocking curve for the reflection from $\mathrm{KI}$ (200) plane. (c) Electron diffraction pattern from a 50-nm-thick KI film.

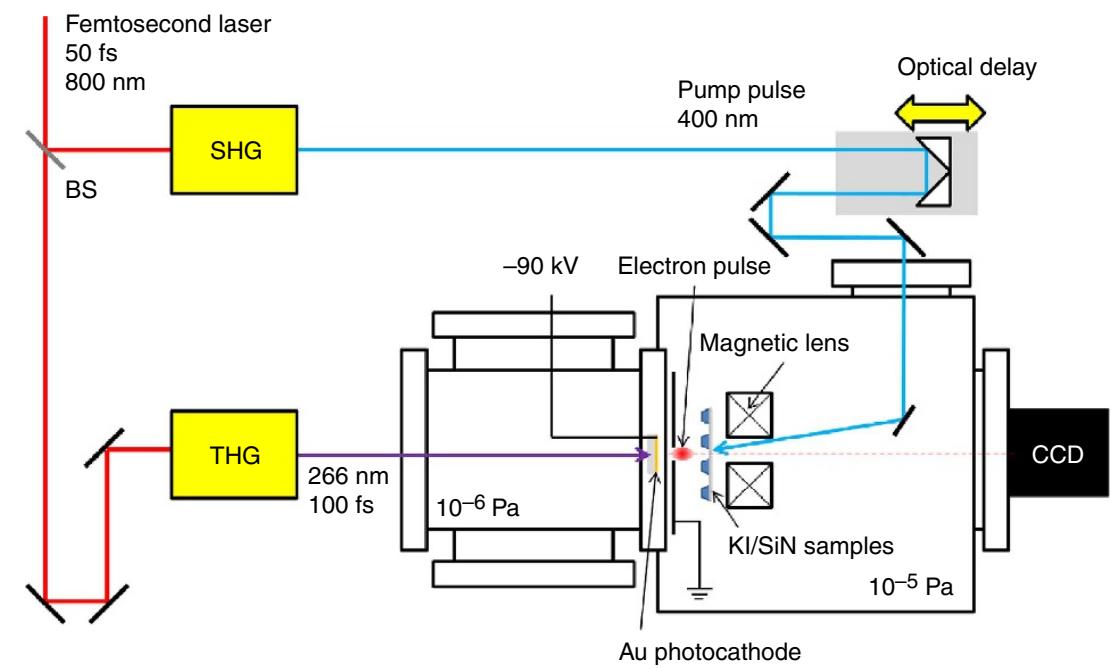

Figure 6 | Schematic diagram of the FED setup. BS, beam splitter; CCD, charge-coupled device camera; SHG, second harmonic generation; THG, third harmonic generation. 
substantial ablation, ion detection thresholds indicate that the sample is likely ejected in the form of large clusters and/or particulates, provided the yield of relatively high (up to 4,000 ) $\frac{\mathrm{m}}{z}$ ions being emitted from the irradiated alkali halide crystals. Such behaviour is common for the fs laser ablation of dielectrics and has been extensively used in industry for pulsed-laser deposition of thin films ${ }^{9}$. The long time scales for the material expulsion in alkali halides nicely correlate with the slow speed of the nanosized particles. However, the assumption about plume composition has to be verified by future experiments.

In summary, we found evidence for a cold laser ablation process that takes place in alkali halides upon photoexcitation of only a few percent of the halide centres. The observed dynamics illustrates the very nature of highly localized repulsive forces involved in the ejection of material. As computing power grows and different simulation tools are developed ${ }^{57}$, ab initio molecular dynamics will be able to provide a more detailed microscopic understanding of this cold ablation process.

\section{Methods}

Single-shot FED measurements. Time-resolved electron diffraction measurements have been performed in transmission mode to minimize the effects of surface charging, which can dominate the behaviour of the electron diffraction signal if reflection mode is used instead ${ }^{58-65}$. The schematic layout of our compact DC-accelerated electron diffraction setup is shown in Fig. 6. A part of the fundamental $(800 \mathrm{~nm}, 50 \mathrm{fs})$ output beam from a commercial Ti:sapphire laser system (Coherent) was split into two arms by a beam splitter. Infrared optical pulses were converted to $400 \mathrm{~nm}$ pump and $266 \mathrm{~nm}$ probe radiation through the second and third harmonic generation nonlinear processes, respectively. The pump light was focused down to a $450-\mu \mathrm{m}$ spot size at the KI sample, while the $266 \mathrm{~nm}$ probe was directed onto a gold photocathode generating ultrashort electron bunches. Photoelectrons were finally accelerated to $90 \mathrm{keV}$ by a DC electric field between the photocathode and anode plates. Photoinduced structural changes inside the material were followed with $\sim 1$ ps pulses containing $\sim 10^{5}$ electrons confined to a $120-\mu \mathrm{m}$ spot in diameter at the ultrathin KI film (vide infra Sample preparation section below). Diffracted and directly transmitted electrons were focused with the magnetic lens onto a 1:1 fibre-coupled CCD camera (Quad-RO 4320, Princeton Instruments) coated with a P20 phosphor scintillator. The time delay between the photoexcitation and electron pulses was adjusted by changing the optical pump-probe path difference using a motorized translational stage. For single-shot measurements, samples were moved between excitation pulses so that each pump pulse would hit a fresh spot. In contrast to previous studies ${ }^{66,67}$ on alkali halide materials using high energy (500-600 keV) and high fluence electrons, no structural changes in KI samples were observed during electron beam irradiation with more than 200 consecutive $90 \mathrm{keV}$ electron pulses.

Single-shot fs optical reflectivity measurements. Single-shot optical pump probe experiments were performed in reflection mode on single-crystalline $\mathrm{Si}$, $\mathrm{NaCl}, \mathrm{CsI}$ and polycrystalline KI films. All samples were photoexcited with $400 \mathrm{~nm}$ $(3.1 \mathrm{eV})$ and $70 \mathrm{fs}$ optical pump pulses focused on the target at an incident angle of $30^{\circ}$ from the surface normal. Different degrees of photoexcitation conditions were achieved by varying the incident pump laser fluence in the $35-1,100 \mathrm{~mJ} \mathrm{~cm}^{-2}$ range. The probe infrared $(800 \mathrm{~nm}, 50 \mathrm{fs}$ ) or white-light pulses (see Supplementary Fig. 5) were focused within the photoexcited area of the sample at a near-Brewster's incident angle of $60^{\circ}$. In the course of this experiment, pump and probe laser beams were $s$ - and $p$-polarized, respectively. The reflected signal intensities were registered with a slow photodiode $(\sim 100 \mu$ s response time) for the infrared probe or using an optical spectrometer (Avantes) when the dynamics were studied with the white-light probe.

Single-shot ion detection measurements. A simple ion detection system was built to determine the relative ion yield as a function of the incident laser fluence. The system is composed of an extraction electrode aperture with either negative or positive polarity at $2.5 \mathrm{kV}$ with respect to ground potential, a drift tube of $200 \mathrm{~mm}$ and a Faraday cup, inside a vacuum chamber. The ions are generated by incident $s$-polarized 400-nm optical pulses, extracted by the electrode and detected by the Faraday cup. The reflection of the incident $400-\mathrm{nm}$ pulses from the samples is used as a trigger for the ion measurements. An $I-V$ converter is used to record the timeof-flight ion signal in an oscilloscope. The sample is translated between laser shots to make sure that a fresh sample area is exposed to each laser excitation pulse; that is, single-shot photoexcitation conditions were used.

Sample preparation. Polycrystalline 50-nm- and 100-nm-thick KI films were prepared by thermal deposition on freestanding silicon nitride windows $(30 \mathrm{~nm}$ thick), and coated with a very thin $(<5 \mathrm{~nm})$ carbon layer to protect the KI films from moisture. Thicker and uncoated, $2.5-\mu \mathrm{m}$-thick KI films were also prepared and used for time-resolved optical reflectivity and crater depth measurements. The deposition rate and film thickness were monitored in situ with a quartz oscillator. Freestanding silicon nitride windows were produced using conventional anisotropic chemical etching methods. The sample crystallinity of KI films was characterized with static X-ray diffraction and electron diffraction. Figure 7a shows the X-ray diffraction spectrum from the KI sample. The diffraction lines from KI (200) and (400) lattice planes are observed, suggesting that crystallites are longitudinally oriented in the $\langle 100\rangle$ direction. This conclusion is also supported by the rocking curve measurement shown in Fig. $7 \mathrm{~b}$. The single-crystalline $\mathrm{Si}, \mathrm{NaCl}$ and CsI samples were used for the experiments as purchased. Out of numerous attempts to prepare thin crystalline samples from different alkali halides under various deposition conditions, films from KI were the only ones with good crystallinity for single-shot FED experiments. To the best of our knowledge, there are also no commercial suppliers providing single-crystalline KI samples.

Computational method. The potential energy curves of the lowest two $1 \Sigma^{+}$states of the $\mathrm{NaCl}$ molecules were calculated using the multi-state, multi-reference CASPT2 (MS-MR-CASPT2) method as implemented in the MOLPRO 2012.1 computational chemistry software package. The cc-pCVDZ and aug-cc-pCVDZ basis sets were used for the metal atoms and the halogen atoms, respectively. For the $\mathrm{NaCl}$ molecule, the active orbitals were: $7 \sigma, 8 \sigma, 9 \sigma, 10 \sigma, 3 \pi_{\mathrm{x}}, 4 \pi_{\mathrm{x}}, 3 \pi_{\mathrm{y}}$ and $4 \pi_{\mathrm{y}}$, that is, an active space of eight electrons in eight orbitals. All orbitals were included in the perturbation calculation.

The potential energy curves are presented in Supplementary Fig. 6. The equilibrium metal-halogen distance on the $1^{1} \Sigma^{+}$curve is $2.421 \AA$. The avoided crossing between the $1^{1} \Sigma^{+}$and $2{ }^{1} \Sigma^{+}$states is found at a distance of $7.8 \AA$.

\section{References}

1. Chichkov, B. N., Momma, C., Nolte, S., Von Alvensleben, F. \& Tünnermann, A Femtosecond, picosecond and nanosecond laser ablation of solids. Appl. Phys. A 63, 109-115 (1996).

2. Liu, X., Du, D. \& Mourou, G. Laser ablation and micromachining with ultrashort laser pulses. IEEE J. Quant. Electron. 33, 1706-1716 (1997).

3. Jeschke, H. O., Garcia, M. E. \& Bennemann, K. H. Theory for the ultrafast ablation of graphite films. Phys. Rev. Lett. 87, 015003 (2001).

4. Miller, J. C. \& Haglund, Jr R. F. Laser Ablation and Desorption 1st edn (Academic Press, 1998)

5. Lorazo, P., Lewis, L. J. \& Meunier, M. Short-pulse laser ablation of solids: from phase explosion to fragmentation. Phys. Rev. Lett. 91, 225502 (2003).

6. Demaske, B. J., Zhakhovsky, V. V., Inogamov, N. A. \& Oleynik, I. I. Ablation and spallation of gold films irradiated by ultrashort laser pulses. Phys. Rev. B 82, 064113 (2010).

7. Zhao, X. \& Shin, Y. C. Coulomb explosion and early plasma generation during femtosecond laser ablation of silicon at high laser fluence. J. Phys. D Appl. Phys. 46, 335501 (2013).

8. Stuart, B. C., Feit, M. D., Rubenchik, A. M., Shore, B. W. \& Perry, M. D. Laser-induced damage in dielectrics with nanosecond to subpicosecond pulses. Phys. Rev. Lett. 74, 2248-2251 (1995).

9. Mao, S. S. et al. Dynamics of femtosecond laser interactions with dielectrics. Appl. Phys. A Mater. Sci. Process. 79, 1695-1709 (2004).

10. Balling, P. \& Schou, J. Femtosecond-laser ablation dynamics of dielectrics: basics and applications for thin films. Rep. Prog. Phys. 76, 036502 (2013).

11. Goldstein, IV E. Arbeiten der wissenschaftlichen Gäste der Abtheilung I. Professor Goldstein's Untersuchungen über Kathodenstrahlen. Zeits. $f$. Instrumentenk. 16, 211 (1896).

12. Hersh, H. N. Proposed excitonic mechanism of color-center formation in alkali halides. Phys. Rev. 148, 928-932 (1966).

13. Rabinowitch, E. Electron transfer spectra and their photochemical effects. Rev. Mod. Phys. 14, 112-131 (1942).

14. Song, K. S., Leung, C. H. \& Williams, R. T. A theoretical basis for the Rabin-Klick criterion in terms of off-centre self-trapped-exciton relaxation. J. Phys. Condens. Matter 1, 683-687 (1989).

15. Tokizaki, T. et al. Femtosecond cascade-excitation spectroscopy for nonradiative deexcitation and lattice relaxation of the self-trapped exciton in NaCl. Phys. Rev. Lett. 67, 2701-2704 (1991).

16. Edamatsu, K., Sumita, M., Hirota, S. \& Hirai, M. Transient absorption due to self-trapped excitons in NaBr and NaI. Phys. Rev. B 47, 6747-6750 (1993).

17. Iwai, S. et al. One-center small polarons as short-lived precursors in selftrapping processes of holes and electron-hole pairs in alkali iodides. Phys. Rev. Lett. 76, 1691-1694 (1996).

18. Shluger, A. L. \& Tanimura, K. Laser-induced reactions in crystals: Femtosecond pump-probe spectroscopy and ab initio calculations of self-trapped excitons and holes in KBr. Phys. Rev. B 61, 5392-5402 (2000).

19. Tanimura, K. \& Hess, W. P. Temperature-dependent yield of Frenkel pairs generated by valence excitation in NaCl. Phys. Rev. B 69, 155102 (2004).

20. Ichimura, N., Kawai, T. \& Hashimoto, S. F-center formation in KI crystals under high-density optical excitation. Phys. Rev. B 75, 155121 (2007). 
21. Dickinson, J. T., Orlando, S., Avanesyan, S. M. \& Langford, S. C. Color center formation in soda lime glass and $\mathrm{NaCl}$ single crystals with femtosecond laser pulses. Appl. Phys. A Mater. Sci. Process. 79, 859-864 (2004).

22. Henyk, M., Costache, F. \& Reif, J. Femtosecond laser ablation from sodium chloride and barium fluoride. Appl. Surf. Sci. 186, 381-384 (2002).

23. Joly, A. G., Hess, W. P., Beck, K. M. \& Dickinson, J. T. Femtosecond time-resolved photo-stimulated desorption from ionic crystals. Appl. Surf. Sci. 186, 339-344 (2002).

24. Hess, W. P., Joly, A. G., Beck, K. M., Williams, R. M. \& Dickinson, J. T. Femtosecond time-resolved laser-induced desorption of positive ions from MgO. Appl. Phys. A Mater. Sci. Process. 69, S389-S393 (1999).

25. Hess, W. P. et al. Laser control of desorption through selective surface excitation. J. Phys. Chem. B 109, 19563-19578 (2005).

26. Kolodziej, J. J. et al. Frenkel defect interactions at surfaces of irradiated alkali halides studies by non-contact atomic-force microscopy. Surf. Sci. 482-485, 903-909 (2001).

27. Stoian, R. et al. Surface charging and impulsive ion ejection during ultrashort pulsed laser ablation. Phys. Rev. Lett. 88, 097603 (2002).

28. Stoian, R., Ashkenasi, D., Rosenfeld, A. \& Campbell, E. E. B. Coulomb explosion in ultrashort pulsed laser ablation of $\mathrm{Al}_{2} \mathrm{O}_{3}$. Phys. Rev. B 62 , 13167-13173 (2000).

29. Shank, C. V., Yen, R. \& Hirlimann, C. Time-resolved reflectivity measurements of femtosecond-optical-pulse-induced phase transitions in silicon. Phys. Rev. Lett. 50, 454-457 (1983).

30. Tom, H. W. K., Aumiller, G. D. \& Brito-Cruz, C. H. Time-resolved study of laser-induced disorder of Si surfaces. Phys. Rev. Lett. 60, 1438-1441 (1988).

31. Sokolowski-Tinten, K., Bialkowski, J. \& Von der Linde, D. Ultrafast laser-induced order-disorder transitions in semiconductors. Phys. Rev. B 51, 14186-14198 (1995).

32. Sokolowski-Tinten, K. et al. Transient states of matter during short pulse laser ablation. Phys. Rev. Lett. 81, 224-227 (1998).

33. Sokolowski-Tinten, K. \& Von der Linde, D. Generation of dense electron-hole plasmas in silicon. Phys. Rev. B 61, 2643-2650 (2000).

34. Choi, T. Y. \& Grigoropoulos, C. P. Plasma and ablation dynamics in ultrafast laser processing of crystalline silicon. J. Appl. Phys. 92, 4918-4925 (2002).

35. Dachraoui, H. \& Husinsky, W. Thresholds of plasma formation in silicon identified by optimizing the ablation laser pulse form. Phys. Rev. Lett. 97, 107601 (2006).

36. Stampfli, P. \& Bennemann, K. H. Time dependence of the laser-induced femtosecond lattice instability of Si and GaAs: Role of longitudinal optical distortions. Phys. Rev. B 49, 7299-7305 (1994).

37. Lindenberg, A. M. et al. Atomic-scale visualization of inertial dynamics. Science 308, 392-395 (2005).

38. Siders, C. W. et al. Detection of nonthermal melting by ultrafast X-ray diffraction. Science 286, 1340-1342 (1999).

39. Rousse, A. et al. Nonthermal melting in semiconductors measured at femtosecond resolution. Nature 410, 65-68 (2001).

40. Harb, M. et al. Electronically driven structure changes of Si captured by femtosecond electron diffraction. Phys. Rev. Lett. 100, 155504 (2008).

41. Zijlstra, E. S., Walkenhorst, J. \& Garcia, M. E. Anharmonic noninertial lattice dynamics during ultrafast nonthermal melting of InSb. Phys. Rev. Lett. 101, 135701 (2008).

42. Cavalleri, A., Sokolowski-Tinten, K., Bialkowski, J., Schreiner, M. \& Von der Linde, D. Femtosecond melting and ablation of semiconductors studied with time of flight mass spectroscopy. J. Appl. Phys. 85, 3301-3309 (1999).

43. Kruer, W. L. Laser plasma interactions with intensities from $10^{12}-10^{21} \mathrm{~W} / \mathrm{cm}^{2}$. Phys. Plasmas 10, 2087-2092 (2003).

44. Favre, C. et al. White-light nanosource with directional emission. Phys. Rev. Lett. 89, 035002 (2002).

45. Von der Linde, D. \& Schüler, H. Breakdown threshold and plasma formation in femtosecond laser-solid interaction. J. Opt. Soc. Am. B 13, 216-222 (1996).

46. Ashkenasi, D., Rosenfeld, A., Varel, H., Wähmer, M. \& Campbell, E. E. B. Laser processing of sapphire with picosecond and sub-picosecond pulses. Appl. Surf. Sci. 120, 65-80 (1997).

47. Gao, H. X., Peng, L.-M. \& Zuo, J. M. Lattice dynamics and Debye-Waller factors of some compounds with the sodium chloride structure. Acta Crystallogr. A 55, 1014-1025 (1999).

48. Peng, L.-M. Quasi-dynamical electron diffraction-a kinematic type of expression for the dynamical diffracted-beam amplitudes. Acta Crystallogr. A 56, 511-518 (2000).

49. Ligges, M. et al. Transient (000)-order attenuation effects in ultrafast transmission electron diffraction. J. Appl. Phys. 109, 063519 (2011).

50. Makinson, J. D. et al. X-ray diffraction signatures of defects in nanocrystalline materials. Adv. X Ray Anal. 42, 407-411 (1998).

51. Wu, S., Geiser, P., Jun, J., Karpinski, J. \& Sobolewski, R. Femtosecond optical generation and detection of coherent acoustic phonons in GaN single crystals. Phys. Rev. B 76, 085210 (2007).
52. Hudert, F. et al. Confined longitudinal acoustic phonon modes in free-standing Si membranes coherently excited by femtosecond laser pulses. Phys. Rev. B 79, 201307(R) (2009).

53. Hayashi, Y. et al. Acoustic pulse echoes probed with time-resolved X-ray triple-crystal diffractometry. Phys. Rev. Lett. 96, 115505 (2006).

54. Tang, J. Coherent phonon excitation and linear thermal expansion in structural dynamics and ultrafast electron diffraction of laser-heated metals. J. Chem. Phys. 128, 164702 (2008).

55. Harb, M. et al. Excitation of longitudinal and transverse coherent acoustic phonons in nanometer free-standing films of (001) Si. Phys. Rev. B 79, 094301 (2009).

56. Moriena, G., Hada, M., Sciaini, G., Matsuo, J. \& Miller, R. J. D. Femtosecond electron diffraction: preparation and characterization of (110)-oriented bismuth films. J. Appl. Phys. 111, 043504 (2012).

57. Zijlstra, E. S., Kalitsov, A., Zier, T. \& Garcia, M. E. fractional diffusion in silicon. Adv. Mater. 25, 5605-5608 (2013).

58. Sciaini, G. \& Miller, R. J. D. Femtosecond electron diffraction: heralding the era of atomically resolved dynamics. Rep. Prog. Phys. 74, 096101 (2011).

59. Park, H. \& Zuo, J. M. Direct measurement of transient electric fields induced by ultrafast pulsed laser irradiation of silicon. Appl. Phys. Lett. 94, 251103 (2009).

60. Okano, Y., Hironaka, Y., Kondo, K.-I. \& Nakamura, K. G. Electron imaging of charge-separated field on a copper film induced by femtosecond laser irradiation. Appl. Phys. Lett. 86, 141501-141503 (2005).

61. Hebeisen, C. T. et al. Direct visualization of charge distributions during femtosecond laser ablation of a Si(100) surface. Phys. Rev. B 78, 081403(R) (2008).

62. Raman, R. K., Tao, Z., Han, T.-R. \& Ruan, C.-Y. Ultrafast imaging of photoelectron packets generated from graphite surface. Appl. Phys. Lett. 95, 181108 (2009).

63. Park, H. \& Zuo, J.-M. Comment on 'structural preablation dynamics of graphite observed by ultrafast electron crystallography'. Phys. Rev. Lett. 105, 059603 (2010).

64. Carbone, F., Baum, P., Rudolf, P. \& Zewail, A. H. Carbone et al. Reply. Phys. Rev. Lett. 105, 059604 (2010).

65. Raman, R. K. et al. Direct observation of optically induced transient structures in graphite using ultrafast electron crystallography. Phys. Rev. Lett. 101, 077401 (2008).

66. Williams, R. T. \& Kabler, M. N. Excited-state absorption spectroscopy of self-trapped excitons in alkali halides. Phys. Rev. B 9, 1897-1907 (1974).

67. Karasawa, T. \& Hirai, M. Color center formation in $\mathrm{KI}$ and $\mathrm{NaCl}$ crystals by pulsed electron beam. J. Phys. Soc. Jpn 39, 999-1003 (1975).

68. Freund, J., Ingalls, R. \& Crozier, E. D. Extended x-ray-absorption fine-structure study of alkali-metal halides under high pressure. Phys. Rev. B 43, 9894-9905 (1991).

\section{Acknowledgements}

We would like to thank Dr K. Sokolowski-Tinten at the University of Duisburg-Essen for helpful discussions, Dr D. Mazurenko and S. Jangam at the University of Hamburg and A. Fox at the University of Toronto for the initial design of the FED setup, A. Casandruc at the University of Hamburg for his help during the electron diffraction experiments and M. Urano at Kyoto University for his help in the determination of the crater depths. This work was funded by the Max Planck Society. M.A.K. acknowledges the Centre for Numerical Algorithms and Intelligent Software (NAIS) for the award of a Principal's Career Development Scholarship. Edinburgh researchers (M.A.K., D.A.W. and C.A.M.) acknowledge the resources of the EPSRC UK National Service for Computational Chemistry Software (NSCCS).

\section{Authors contributions}

R.J.D.M. is principle investigator for this project. R.J.D.M., M.H. and G.S. conceived and coordinated this project. D.Z. developed the optical experimental setup. J.H., S.A.H., S.M., R.Y.N.G., D.Z., M.H., G.M. and G.S. developed the electron diffraction system. M.H., D.Z. and R.Y.N.G. developed the ion detection experimental setup. D.Z. and K.P. automated the setups. M.H. and D.Z. performed the optical, ion detection and FED experiments. M.H. made the thin alkali halide samples. J.M. and T.S. performed the interferometric measurements. M.A.K., D.A.W. and C.A.M. calculated the potential surface energy of alkali halide molecules. M.H., D.Z., K.P., G.S. and R.J.D.M. interpreted the data. M.H., D.Z., K.P., G.S. and R.J.D.M. wrote this manuscript with helpful suggestions from all co-authors.

\section{Additional information}

Supplementary Information accompanies this paper at http://www.nature.com/ naturecommunications

Competing financial interests: The authors declare no competing financial interests

Reprints and permission information is available online at http://npg.nature.com/ reprintsandpermissions/

How to cite this article: Hada, M. et al. Cold ablation driven by localized forces in alkali halides. Nat. Commun. 5:3863 doi: 10.1038/ncomms4863 (2014). 